\title{
Léontine Zanta, philosophe et romancière : notes sur le parcours d'une pionnière
}

Léontine Zanta, filosofa e scrittrice: appunti sul percorso di una pioniera Léontine Zanta, Philosopher and Novelist: Notes on a Pioneer's Career

Annabelle Bonnet

\section{(2)enEdition}

Journals

Édition électronique

URL : https://journals.openedition.org/cei/8780

DOI : $10.4000 /$ cei.8780

ISSN : 2260-779X

Éditeur

UGA Éditions/Université Grenoble Alpes

Édition imprimée

ISBN : 978-2-37747-257-4

ISSN : $1770-9571$

Référence électronique

Annabelle Bonnet, « Léontine Zanta, philosophe et romancière : notes sur le parcours d'une pionnière », Cahiers d'études italiennes [En ligne], 32 | 2021, mis en ligne le 01 mars 2021, consulté le 17 septembre 2021. URL : http://journals.openedition.org/cei/8780; DOI : https://doi.org/10.4000/cei 8780

Ce document a été généré automatiquement le 17 septembre 2021. 


\title{
Léontine Zanta, philosophe et romancière : notes sur le parcours d'une pionnière
}

\author{
Léontine Zanta, filosofa e scrittrice: appunti sul percorso di una pioniera \\ Léontine Zanta, Philosopher and Novelist: Notes on a Pioneer's Career
}

\section{Annabelle Bonnet}

\section{Introduction}

1 Qui se souvient du nom de celle qui inspira Simone de Beauvoir dans sa volonté d'étudier la philosophie à une époque où cette activité était encore réservée aux hommes? La philosophe la mentionne pourtant explicitement dans ses mémoires, lorsqu'elle explique son choix de se tourner vers des études de philosophie :

J'avais lu dans une revue un article sur une femme philosophe qui s'appelait Mlle

Zanta: elle avait passé son doctorat [...]. Les femmes qui avaient alors une agrégation ou un doctorat de philosophie se comptaient sur les doigts de la main : je souhaitais être une de ces pionnières ${ }^{1}$.

2 L'objectif du présent article, réalisé à partir de recherches effectuées pour une thèse de doctorat portant sur l'accès des femmes à la philosophie en France depuis la fin du XIX siècle ${ }^{2}$, est de revenir sur le parcours impressionnant de cette pionnière qu'a été Léontine Zanta, première femme française docteure en philosophie ${ }^{3}$. Le terme de pionnière est entendu afin de désigner ces femmes qui, grâce à leurs diplômes et réussites scolaires, pénètrent dans des milieux professionnels masculins et contreviennent aux normes sociales de leurs époques, tout en ne disposant « d'aucun modèle antérieur ${ }^{4}$ » féminin auquel s'identifier. Plus particulièrement, l'article entend examiner cette figure autour du prisme de sa relation avec la littérature : car, si c'est bien le succès philosophique qui la porte d'abord au statut de pionnière, c'est ensuite sous la forme littéraire, notamment les romans, que Léontine Zanta poursuit son œuvre. Cet article explore ce parcours au prisme de trois dimensions : d'une part, celle 
du succès mais aussi des difficultés de légitimité rencontrées par une femme philosophe à cette époque, qui ne dispose pas de statut professionnel et se tourne alors vers les réseaux de femmes de lettres. D'autre part, celle de la création du personnage de l'étudiante de philosophie dans ses romans, figure historiquement nouvelle et qu'elle met en scène à deux reprises, ainsi que celle des destins qu'elle offre à ce personnage, qui poussent les lectrices de l'époque vers une conclusion explicitement développée: les femmes ne réussissent leur vie morale et sociale que lorsqu'elles abandonnent les études philosophiques et la prétention d'être des philosophes. Conclusion pessimiste des romans de Léontine Zanta à laquelle on proposera, pour finir, une explication, au prisme de sa conception de la vie sociale et de ses positions politiques.

\section{Une philosophe médiatique}

3 Léontine Zanta est la première femme de nationalité française à obtenir un doctorat de philosophie en France, en mai 1914. C'est alors un véritable événement médiatique, comme en témoignent les divers articles couvrant l'événement ${ }^{5}$. Dans un entretien retrouvé, elle décrit elle-même le jour de sa soutenance en ces mots :

C'était le 19 mai 1914, à quelques mois de la guerre. Jamais la salle de soutenance à la Sorbonne n'avait connu pareille affluence [...]. Et puis le succès final, les applaudissements, l'apothéose! En sortant, la ruée des photographes et des journalistes: j'étais une vraie star, l'étoile de la Sorbonne! Et le lendemain ma personne suspendue en image dans tous les kiosques de journaux et mon avenir assuré par des conférences dans toute l'Europe ${ }^{6}$ !

4 Le jury de thèse est alors composé de philosophes célèbres de l'époque: Fortunat Strowski, Lucien Lévy-Bruhl, Victor Delbos, François Picavet, mais aussi Gabriel Séailles qui est le président du jury. Désirant prendre part à un renouveau du catholicisme alors en perte d'influence durant cette période de sécularisation ${ }^{7}$, son travail porte sur les rapports entre stoïcisme et christianisme et les bienfaits du premier pour revivifier le second ${ }^{8}$. Mais la popularité de cet événement provient surtout de l'exploit réalisé par Léontine Zanta. Il faut en effet rappeler que Léontine Zanta naît en 1872, obtient sa licence de philosophie en 1898, à 26 ans, et son doctorat en philosophie en 1914, à 42 ans. Sa formation va à contretemps, puisqu'elle se déroule dans un moment où l'accès des femmes à l'enseignement philosophique public en France est presque impossible.

De fait, la philosophie est entrée, depuis le $\mathrm{xx}^{\mathrm{e}}$ siècle, dans un grand processus de transformation, passée d'une pratique culturelle non définie en termes scolaires et institutionnels à une discipline scolaire réglementée par le ministère de l'Instruction publique autour de programmes officiels, de diplômes et de concours publics d'accès au professorat. Sous la Troisième République, elle devient tout particulièrement la " discipline de couronnement ${ }^{9}$ » des études secondaires des programmes des garçons. Pourtant, la loi Camille Sée, qui organise, elle, l'enseignement secondaire public pour les filles, ne prévoit aucun enseignement de philosophie, au prétexte qu'un homme ne souhaite, en rentrant d'une journée de travail, "trouver sa femme observant les astres $^{10}$ ». Une des spécificités de la discipline en cette période est de résister à l'entrée des femmes et d'associer "masculinité et puissance théorique ${ }^{11}$ ». Il n'existe donc pas de classe de philosophie dans le secondaire pour les jeunes filles telle qu'elle est établie pour les garçons, ce qui leur rend difficile un potentiel accès à l'ensemble des titres 
nécessaires pour être qualifié de philosophe: le baccalauréat, dont la philosophie constitue une discipline centrale, mais aussi la licence, tout comme l'agrégation de philosophie, manquent alors. Léontine Zanta s'intègre donc dans un espace qui lui est réfractaire du point de vue de la loi.

Il faut toutefois mentionner que Léontine Zanta n'est pas la première femme docteure en philosophie en France, puisqu'il s'agit d'Alice Steriad, philosophe roumaine devenue docteure en 1913 et dont on ne trouve par ailleurs aucune mention faite de la part de Léontine Zanta ni de la presse et des revues féministes de l'époque ${ }^{12}$. Car Léontine Zanta tire aussi son prestige et attire l'attention de la presse et des milieux intellectuels, dans une période de fort nationalisme d'avant-guerre, par son origine alsacienne comme par la popularité de son père dans les milieux universitaires. On rappelle en effet volontiers que "mademoiselle Zanta a de qui tenir: elle est la fille d'un professeur de Colmar demeuré Français après $1870^{13}$ ». Elle est en effet née à Mâcon, dans une famille alsacienne qui s'est exilée et est aussi profondément catholique. Son père, Charles Zanta, est un professeur agrégé de grammaire, connu pour ses positions politiques en défense de la France. C'est aussi grâce à lui que Léontine Zanta échappe à une éducation plus traditionnelle de petite fille, racontant elle-même que celui-ci éprouvait le désir d'avoir un fils avant d'ajouter avec humour : «Ce fils fut une fille : moi ! Ceci l'incita à m'élever comme un garçon ${ }^{14}$. » Enfin, c'est lui qui l'initie aux langues anciennes et à la philosophie, jusqu'au jour où son ami, le philosophe Gabriel Séailles, propose à Léontine Zanta d'assister à ses cours de philosophie ${ }^{15}$. Elle obtient par la suite son baccalauréat et part à Paris étudier la philosophie à la Sorbonne, section où elle sera la seule fille durant toute sa formation. Elle obtient ensuite sa licence de philosophie en 1898 , puis développe sa carrière au sein de l'enseignement privé ${ }^{16}$.

\section{Le passage vers la littérature et le personnage de l'étudiante en philosophie}

7 En 1917, Léontine Zanta reçoit le prix Marcelin Guérin de l'Académie française pour la publication de sa thèse ${ }^{17}$, ce qui la promet à un bel avenir. Pourtant, après son succès au doctorat, elle ne développe pas sa carrière au sein de l'espace philosophique universitaire. Elle continue en effet de donner des cours au lycée. Notons qu'après le doctorat, on ne lui propose aucun emploi à l'université, mais que son ambition était pourtant bien d'occuper une chaire de philosophie ${ }^{18}$. Notons aussi que l'occupation d'une chaire de philosophie pour une femme en France n'arrivera pour la première fois qu'après la seconde guerre mondiale.

Léontine Zanta poursuit alors ses travaux au sein de l'espace journalistique, en écrivant pour des journaux à grand tirage comme L'Écho de Paris, journal où elle publie en première page, mais aussi Le Correspondant, Le Figaro, La Revue hebdomadaire, pour lesquels on comptabilise plus de 200 articles à son actif. Elle développe aussi ses écrits au sein de l'espace littéraire, tout particulièrement au contact d'un réseau de femmes de lettres en pleine constitution: celui du jury Femina, dont elle devient membre en 1920. Ce prix a été conçu afin de favoriser la reconnaissance littéraire des femmes et leur capacité de création. Il rassemble des femmes issues des milieux de la bourgeoisie, mais qui se situent à la marge du champ littéraire, puisqu'il a été fondé en 1904 pour contrer l'hostilité faite aux femmes au sein du jury Goncourt ${ }^{19}$. Léontine Zanta publie 
par ailleurs dans le journal féministe La Française, dont elle partage les positions concernant le droit de vote des femmes et l'égalité d'accès aux carrières libérales.

Mais c'est surtout comme romancière que Léontine Zanta va développer son œuvre: quatre de ses cinq livres publiés sont en effet des romans. Dans les années 1920, elle écrit La science et l'amour, journal d'une étudiante et publie en 1927 La part du feu, pour lequel elle reçoit le prix de littérature spiritualiste ${ }^{20}$. Puis elle rédige Sainte Odile, paru en 1931 et Sainte Monique et son fils en 1941, avant sa mort. Durant la première partie du $\mathrm{xx}^{\mathrm{e}}$ siècle, il faut noter que l'espace littéraire pour les femmes auteures s'élargit, même si cette extension ne se fait pas au centre du champ littéraire, fortement réfractaire à leur présence ${ }^{21}$. Il faut aussi noter la constitution, avec l'industrialisation du livre et la libéralisation économique, d'un marché des biens symboliques autour des romans, qui peut expliquer la venue en littérature de nombreuses femmes, d'autant qu'il existe désormais un public féminin qui est en demande de lectures ${ }^{22}$.

Ses deux premiers livres sont des romans d'apprentissage. Si la figure de l'étudiante est un thème toujours plus présent en littérature à cette période ${ }^{23}$, les romans de Zanta s'emparent d'une nouveauté dans les personnages littéraires, qui est aussi une nouvelle figure sociale : la figure de l'étudiante en philosophie. La science et l'amour et La part du feu mettent ainsi tous les deux en scène des femmes philosophes. Dans le premier roman, on suit les expériences de Madeleine, étudiante de première année en philosophie à la Sorbonne. Dans le second, on accompagne la vie de Sabine alors qu'elle est déjà licenciée de philosophie. Les deux ouvrages sont centrés autour d'une question similaire et plutôt classique : comment, pour une femme, concilier intellect et amour ? Comment ne pas perdre sa féminité et ses qualités de femme? Cette opposition proposée par la Léontine Zanta n'a rien d'étonnant d'un point de vue historique: Françoise Blum a déjà montré qu'il est commun que les mouvements féministes de la Troisième République problématisent les questions de la professionnalisation des femmes sous cet angle ${ }^{24}$.

11 Ainsi, Madeleine administre ses études et les travaux domestiques classiques à effectuer chez sa famille et est à la recherche d'un équilibre entre ces deux sphères. Mais Madeleine s'ennuie rapidement à la Sorbonne, dont les études, tournées vers le positivisme, sont présentées comme lui faisant perdre le sens de la vie. C'est alors sa rencontre avec un autre étudiant qui va peu à peu prendre de l'importance dans sa vie : étudiant plus âgé, ils se fréquentent alors que Madeleine émet d'abord le souhait de rester célibataire et de se consacrer à ses études, avant de se rétracter et d'accepter un mariage avec lui. Puis, l'étudiant finit par la quitter avant que les noces n'aient lieu. Madeleine, perdue intellectuellement et sentimentalement, rate alors ses examens et se retrouve seule, tandis que l'étudiant les réussit et poursuit sa route. Madeleine n'arrive ni à administrer ses sentiments, ni à finir ses études. Elle ne parvient pas à surmonter les deux pôles de sa vie, privé et professionnel, et échoue. Mais c'est à la fin du roman, une fois l'apprentissage terminé, que se révèlent les raisons de sa chute. Léontine Zanta lui fait alors dire :

N'est-ce point-là le danger, l'écueil de notre formation trop exclusivement intellectuelle ? Notre sensibilité est trop longtemps refoulée, contenue, et le jour où elle s'éveille nous ne savons pas la conduire, faute d'y avoir été préparées ${ }^{25}$.

Elle compare aussi les femmes philosophes à des femmes manquées : "Nous devrions être des femmes, et nous raisonnons comme des hommes, l'égoïsme en moins, des sentiments de tendresse dont nous détruisons ainsi toute la spontanéité et tout le 
charme ${ }^{26}$ ", affirme-t-elle. C'est une contradiction qui provoque de la souffrance pour Madeleine, qui se lamente alors: "Je me sens entrânée par ma nature sensible de femme, et mon cerveau me demande d'être virile. Voilà le secret de tant de troubles, de tant de souffrances ${ }^{27} "$, avant de conclure :

J'ai trop fréquenté les livres et je ne connais pas assez la vie [...]. C'est ce qui explique chez moi, et chez tant d'autres de ma sorte, un certain déséquilibre, une psychologie qui déroute ceux qui nous approchent, comme elle nous déroute souvent de nous-mêmes ${ }^{28}$.

13 À son tour, dans le roman La part du feu, Sabine, licenciée de philosophie, tombe amoureuse d'un médecin marié, qui lui-même se repentit d'avoir été infidèle à sa femme et trouve dans la foi catholique un équilibre qu'il tente d'inculquer aussi à Sabine, dont la psyché a été déstabilisée par ses études de philosophie. Sabine, devenue en effet matérialiste à cause de ses études, surmonte son problème et ne trouve la paix qu'en se livrant à Dieu. La position religieuse catholique prévaut alors sur le dilemme, et l'héroïne doit apprendre à vivre dans le dévouement pour trouver son équilibre.

14 Ainsi, les deux hérö̈nes échouent à être des personnes morales si elles sont trop proches de la philosophie. Et surtout, la philosophie empêche de vivre dignement. Ces dénouements donnés aux héroïnes étonnent lorsqu'on connaît les obstacles surmontés par Léontine Zanta et lorsqu'on connaît aussi son combat pour la dénonciation des préjugés concernant la capacité intellectuelle des femmes. Le pessimisme de Madeleine et la fuite de Sabine du monde philosophique tranchent en effet avec la pratique féministe de Léontine Zanta. À titre d'exemples, en 1923, elle publie dans Le Figaro un article sous le titre «La Femme électeur ${ }^{29}$ ». En 1925, elle rédige un autre article dans le même journal pour y revendiquer à nouveau le droit de vote des femmes ${ }^{30}$. En 1927, elle intervient à la radio sur le thème « Les femmes votaient au Moyen Âge ${ }^{31}$ » et donne une conférence intitulée "L'évolution du droit de la femme ${ }^{32}$ ». Elle participe aussi aux États généraux du féminisme en février $1929^{33}$, qui réunissent diverses associations de femmes françaises en vue de discuter notamment du droit de vote des femmes. Pourtant, Léontine Zanta se replace, dans ses romans au sein de la tradition des femmes liseuses, comme une grande partie de la tradition littéraire depuis le XvIII ${ }^{\mathrm{e}}$ siècle, qui montre la femme lectrice et cultivée en danger, car la lecture, particulièrement la lecture philosophique, corrompt l'éducation féminine et les femmes s'y perdent ${ }^{34}$. Comment alors comprendre ce contraste entre son parcours personnel et sa représentation de la vie de femmes étudiant la philosophie?

\section{L'impossible conciliation entre femmes et philosophie}

Une piste pour comprendre la mise en scène de ces échecs féminins peut être donnée à l'éclairage de son travail théorique portant sur le féminisme et sa vision de la société et ses positionnements politiques. Car, malgré le fait de défendre le droit de vote et l'accès des femmes à de nouvelles professions, Léontine Zanta considère en effet que le travail des femmes est positif dans la mesure où, comme elle l'affirme elle-même, il les aide à devenir « de meilleures compagnes pour les hommes ${ }^{35}$ ", objectif principal, selon elle, du savoir pour les femmes. Comme le rappelle l'historienne Françoise Thébaud, la situation des femmes sans droits politiques sous cette période historique est telle qu'elle contraint une partie des féministes existantes «au paradoxe de réclamer 
l'égalité au nom de la différence ${ }^{36}$ ", et sur ce point, les mises en tension proposées par Léontine Zanta ne sont donc pas originales. Dans cette conception en effet, les hommes et les femmes n'ont pas les mêmes capacités physiologiques ni les mêmes rôles sociaux à tenir.

De même, Léontine Zanta, connue pour être une tête d'affiche du féminisme catholique, défend avec rigueur la famille comme principe sacré ainsi que le maintien de la foi pour les femmes. En 1923, elle revendique le droit de vote pour les femmes, mais fustige aussi le féminisme lié au socialisme et se demande si «cette idée confuse que représente le mot féminisme n'a pas contaminé cette autre idée si simple et si claire pourtant du droit de vote ${ }^{37}$ ». La faute serait en effet à « une image disgracieuse »du féminisme, une mémoire collective « hantée par le spectre des premières féministes à l'allure ridicule ${ }^{38}$ ». Elle y oppose cette nouvelle génération de femmes avocates, médecins, agrégées, doctoresses qui n'auraient, selon elle, pas perdu leur féminité. Sa conception de la défense du droit des femmes est par ailleurs longuement développée dans son seul ouvrage théorique, intitulé Psychologie du féminisme ${ }^{39}$. Dans celui-ci, la philosophe définit et oppose deux types de féminisme. Le premier, qu'elle revendique et nomme "féminisme d'adaptation", est défini comme une défense du droit des femmes ne rompant pas avec l'ordre social existant puisqu'il « est orienté par une politique favorable à la famille $e^{40} \%$. Le deuxième, qu'elle rejette, est nommé féminisme de combat, qui se manifeste selon elle lorsque «la femme ne trouve plus sa place dans le milieu social brusquement changé ${ }^{41}$ ", faisant référence aux moments révolutionnaires. Par cette désignation, elle rejette ainsi à la fois le féminisme socialiste, qu'elle combat avec vigueur depuis la Révolution russe, mais aussi le féminisme républicain laïc. La philosophe prend en effet position au sein de ce que l'historienne Florence Rochefort a nommé la "guerre des deux France ${ }^{42}$ ", c'est-à-dire d'une opposition entre féminisme laïque et féminisme catholique.

Il faut rappeler que Christine Bard a déjà souligné que les relations entre ces deux types de féminisme deviennent d'autant plus difficiles à partir des années 1920 avec l'arrivée du cartel des gauches et la montée d'un antisoviétisme fort : des catholiques organisent les mouvements féminins contre le féminisme de manière générale, et contre un catholicisme social qui, parfois, lui est plus favorable, valorisant au contraire la création d'espaces de développement propre aux femmes dans un respect de la foi catholique, du devoir familial et souvent accompagnées d'un fort chauvinisme ${ }^{43}$. De même, durant cette période, si la convergence autour de l'idée que la citoyenneté devient pour les femmes catholiques une étape imposée, ces dernières restent amplement dubitatives quant à la défense d'un modèle de la femme indépendante, préférant défendre le foyer et l'obéissance au «chef de famille ${ }^{44}$ ». Soulignons aussi qu'il en est de même lorsqu'il s'agit de penser la question de l'apparition massive des étudiantes qui visent une profession et une indépendance économique : dès 1912, le cardinal Alfred Baudrillart, recteur de l'Institut catholique de Paris et membre de l'Académie française, publie un article montrant la préoccupation de l'Église face à la quantité de jeunes filles étudiantes. Il affirme ainsi que la Sorbonne et les universités les mettent en "contact de tous les instants avec l'esprit critique ", avec des exposés "sans réserve ni précautions de tous les systèmes » et provoque des rencontres avec " de jeunes étrangères, sans traditions ni principes, anarchistes et révolutionnaires ${ }^{45}$ ». Bruno Poucet a sur ce point mis au jour la stigmatisation de l'enseignement public auprès des catholiques par le Code de droit canonique de 1917, mais aussi les postures 
multiples et contradictoires des catholiques face à la République et à l'école laïque dans l'entre-deux guerres, qui ne peuvent être résumées à une seule tendance ${ }^{46}$.

Dans tous les cas, Léontine Zanta s'inscrit pleinement dans ce cadre réfractaire à l'enseignement public. Dans un article dont le nom est explicite, intitulé « Esprit laïque, esprit de destruction ${ }^{47}$ ", elle accuse l'Instruction publique de voler les enfants aux familles et, selon son terme, de «les briser » et accuse le régime en place de politique «destructive d'une autre morale qui a fait ses preuves puisqu'elle a traversé vingt siècles de civilisation sans qu'on ait pu y porter atteinte ${ }^{48} »$. Elle s'en prend encore à ce qu'elle nomme "la république des camarades ", en référence au nom que les membres du Parti communiste se donnent entre eux, qui lui sert à désigner le régime du Cartel des gauches. Selon elle, les professeurs à la tête de la Sorbonne, particulièrement les professeurs de philosophie, seraient favorables à la politique du Cartel des gauches et iraient jusqu'à manipuler les résultats afin de favoriser des étudiantes et de mieux dérégler la société 49 .

Pour comprendre une telle attaque, il faut rappeler, comme Bruno Poucet l'a montré, que dès les années 1860 , l'enseignement de la philosophie entre dans un processus d'autonomisation de la théologie, qui l'abrite jusqu'alors, pour devenir peu à peu un enseignement relevant du domaine public ${ }^{50}$. Plus encore, les philosophes sont pris dans un long processus entendant placer sous le contrôle de l'État l'Institution scolaire en vue de la formation d'un "esprit public ${ }^{51}$ ", dont une des caractéristiques centrales est de se constituer en se dégageant autant que faire se peut de l'Institution cléricale et de sa main mise sur l'enseignement qui régnait jusqu'à la veille de la Révolution française $\mathrm{e}^{52}$. L'espace philosophique joue par ailleurs un rôle central dans la formation d'une morale républicaine laïque ${ }^{53}$, et c'est dans cette perspective que Léontine Zanta en vient à rejeter un certain enseignement de la philosophie, percevant dans la philosophie universitaire de son temps un ennemi de la religion catholique. Alors qu'en 1914, elle discutait sur ce terrain de la question du christianisme, en 1927, elle va désormais jusqu'à la réfutation en opposant drastiquement la philosophie universitaire de son temps à sa foi catholique: «Assez de philosophes! Je garde ma solution, mais c'est au Christ que je demanderai la formule ${ }^{54}$.»

Il faut enfin noter que ses deux dernières œuvres littéraires, consacrées à sainte Odile et sainte Monique (cette dernière étant publiée sous l'Occupation en 1941), connaissent un sévère fléchissement quant à la question de l'émancipation des femmes ${ }^{55}$. Alors que la dimension combative des femmes existait malgré tout dans le roman Journal d'une étudiante et que le droit à l'égalité intellectuelle et professionnelle était une valeur malgré tout revendiquée par Léontine Zanta, qui n'en prônait pas pour autant un abandon des valeurs familiales, la dimension émancipatrice disparaît complètement de ses deux dernières études. À travers son étude sur les saintes, Léontine Zanta opère en effet une dérive dans la définition même du terme de féminin, désormais à peine entendu à partir du culte du sacrifice à la famille. Toute émancipation hors de celle-ci contient alors un potentiel endommagement envers la société, alors que les femmes sont garantes de l'ordre social. Autrement dit, dans les deux derniers ouvrages, les femmes ne sont simplement plus pensées comme des individus : elles n'existent plus pour elles-mêmes mais uniquement dans une dévotion absolue à la famille, à Dieu et à la patrie.

22 Sa vision du rôle des femmes dans la société est ici toute similaire à celle que Francine Muel-Dreyfus a décrit comme relevant de l'idéologie des femmes sous le régime 
collaborateur de Vichy: un discours principalement articulé autour de l'idée de renoncement pour les femmes en vue de créer une culture du sacrifice devant la patrie et pour favoriser la restauration de valeurs catholiques antirépublicaines qui auraient été bafouées ${ }^{56}$. Ce tournant de Léontine Zanta ne lui est pas propre, mais reflète aussi un fléchissement sociétal et politique d'une partie des intellectuels français nationalistes, antilaïcs et anticommunistes ${ }^{57}$. Camille Cleret a consacré dans ce sens une étude aux femmes catholiques ayant adhéré à l'Action française dans une opposition viscérale aux valeurs républicaines ${ }^{58}$ et Anne Simonin a montré l'adhésion de femmes des œuvres catholiques et sociales au pétainisme sur la base d'options communes qui superposent antisyndicalisme, anticommunisme, idées contre-révolutionnaires et antirépublicanisme, mais aussi service social, défense de la famille et nationalisme ${ }^{59}$.

De fait, Léontine Zanta tient depuis la Première guerre mondiale un salon où se rencontrent les écrivains Paul Bourget, Robert Garric et Maurice Donnay, qui sont tous trois marqués par leur catholicisme et surtout leur proximité au mouvement d'extrême droite l'Action française. Maurice Barrès, plus organique au sein de l'Action française, y est aussi présent ${ }^{60}$. Léontine Zanta s'empare aussi du symbole de Jeanne d'Arc : « Il nous faut des Jeanne d'Arc humanisées, des saintes descendues de leur piédestal, des vierges au regard direct ${ }^{61} "$, affirme-t-elle face au péril que courrait la France. Enfin, après l'élection du Front populaire, Léontine Zanta rédige un article au sein duquel elle fait une longue métaphore filée comparant le siège de Rome à l'invasion communiste, et appuyant ouvertement les mouvements d'extrême droite et le fascisme :

Nous avons des hommes qui agissent dans notre République, qui prêchent l'amour, la réconciliation des classes, qui comprennent qu'il n'est qu'un moyen de sauver la civilisation et la Patrie ; on les traite de factieux, on les accuse de fascisme, cela veut dire, d'après le dictionnaire " fronte populare », de trahison ${ }^{62}$.

Dès 1931, L'Action française s'associe par ailleurs à la pensée de Zanta et n'hésite pas à en valoriser les ouvrages. La revue loue ainsi son livre ayant pour sujet sainte Odile et qui ne cherche pas à « dépeupler la terre en vidant le ciel, à quoi s'emploient farouches partisans du matérialisme marxiste ${ }^{63}$ ». De la même manière, Léontine Zanta commence à parler au nom de "l'avenir de notre art régénéré64 ", et s'approprie ainsi le thème de la régénération de la culture, leitmotiv de l'extrême droite de l'Action française puis de la période de Vichy ${ }^{65}$.

\section{Conclusion}

Ainsi, les romans de Léontine Zanta témoignent d'une tension, au sein de sa pensée, quant à la question de l'accès des femmes à la philosophie. D'un côté, elle prône et loue les avancées des femmes au sein des professions intellectuelles, mais toujours en les subordonnant au maintien de la foi catholique et de ses valeurs. De l'autre, le champ philosophique de son temps, à l'intérieur duquel se développent les romans analysés, est interprété comme un espace dangereux pour les filles, républicain voire révolutionnaire et auquel elle s'oppose de plus en plus drastiquement au cours des années 1920. Pour l'histoire des femmes et du genre, le parcours de Léontine Zanta est donc à considérer en tant qu'il s'agit d'une pionnière, mais il est aussi à examiner à la lumière de ses choix politiques au sein de son contexte historique, qui la mettent devant d'importantes contradictions donnant forme à ses romans. 


\section{NOTES}

1. S. de Beauvoir, Mémoires d'une jeune fille rangée, Paris, Gallimard, 1958, p. 222.

2. A. Bonnet, "La barbe ne fait pas le philosophe». Les femmes et la philosophie en France (1880-1949), thèse soutenue sous la direction de Jean-Louis Fabiani, le 10 janvier 2020, EHESS, Paris. Je remercie par ailleurs Victor Neves, pour ses discussions et suggestions quant à penser le parcours de Léontine Zanta au prisme des problèmes posés par la sociologie des intellectuel(le)s, par rapport à la relation entre permanences et transformations au sein de la formation d'une pensée individuelle.

3. Voir G. Fraisse, La raison des femmes, Paris, Plon, 1992, p. 92. Puis Henri Maleprade, époux de la nièce de Léontine Zanta, lui consacre un ouvrage: H. Maleprade, Léontine Zanta: vertueuse aventurière du féminisme, Paris, Rive droite, 1997. F. Le Guennec, « Léontine Zanta », dans B. Didier, A. Fouque et M. Calle-Grubert (éds), Le dictionnaire universel des créatrices, éditions Des femmes, 2013. C. Bard, "Léontine Zanta ", dans C. Bard et F. Rochefort (éds), Dictionnaire des féministes. France- $\mathrm{XVII}^{e}-\mathrm{XXI} \mathrm{e}^{e}$ siècle, Paris, PUF, 2017. F. Gugelot, "Suzanne Bing, Ève Lavallière, Isabelle Rivière, Léontine Zanta ", dans A. Cova et B. Dumons (éds), Destins de femmes. Religion, culture et société France (XIX ${ }^{e}-X^{e}$ siècles), Paris, Letouzey et Ané, 2010, p. 74-75.

4. G. Fraisse, « Pionnières », Mil neuf cent, $\mathrm{n}^{\circ}$ 16, 1998, p. 7.

5. Voir J. Pain, « Le premier docteur en philosophie », La Française, 30 mai 1914 ; Id., « Une femme doctoresse ès philosophie ", Excelsior, 20 mai 1914, p. 2 ; Id., «La première femme docteur en philosophie ", L'Intransigeant, 20 mai 1914, p. 1 ; Séverine, « La stoïcienne », L'Intransigeant, 28 mai 1914, p. 1.

6. "Mlle Léontine Zanta, la première femme française ayant obtenu le titre de docteur en philosophie pour le grand doctorat d'État ", Les dimanches de la femme: supplément de la mode, 29 décembre 1935, p. 3.

7. Voir M. Gauchet, Le désenchantement du monde. Une histoire politique de la religion, Paris, Gallimard, 1985.

8. L. Zanta, La renaissance du stoïcisme au seizième siècle, Paris, Champion, 1914.

9. J. Fabiani, Les philosophes de la République, Paris, Les Éditions de Minuit, 1988 ; P. Vermeren, Victor Cousin. Le jeu de la philosophie et de l'État, Paris, L'Harmattan, 1995.

10. "Séance du 19 janvier 1880 ", Lycées et collèges de jeunes filles. Documents rapports et discours à la chambre des députés et au Sénat, décrets, arrêtés, circulaires, etc., relatifs à la loi sur l'enseignement secondaire des jeunes filles, Léopold Cerf, Paris, 1888, p. 204. Sur ce point, F. Mayeur, L'enseignement secondaire des jeunes filles sous la Troisième République, Paris, PUF, 1977, p. 33 ; B. Poucet, Enseigner la philosophie. Histoire d'une discipline scolaire (1860-1990), Paris, CNRS Éditions, 1999, p. 140.

11. J. Fabiani, Les philosophes de la République, ouvr. cité, p. 9 ; J. Fabiani, Qu'est-ce qu'un philosophe français? La vie sociale des concepts (1880-1980), Paris, Éditions de l'EHESS, 2010, p. 60.

12. Son exploit passe complètement inaperçu : qu'il s'agisse de L'Action féministe, L'Action féminine, La Française ou encore La Fronde, les revues ne la mentionnent pas. Son travail est pourtant loué par les philosophes. Voir Revue de Métaphysique et de Morale, supplément, mai 1914, p. 9-10.

13. «Échos ", Le Journal, 20 mai 1914, p. 1.

14. "Mlle Léontine Zanta, la première femme française ayant obtenu le titre de docteur en philosophie pour le grand doctorat d'État ", Les dimanches de la femme: supplément de la mode, 29 décembre 1935, p. 3.

15. Comme mentionné dans l'entretien « Mlle Léontine Zanta, la première femme française ayant obtenu le titre de docteur en philosophie pour le grand doctorat d'État », art. cité.

16. « Décret du 12 octobre 1904 », Journal officiel de la République française, 1904, p. 6110. 
17. «Académie française : séance du 12 juillet, présidence de Frédéric Masson », Journal officiel de la République française, 1917, p. 5612.

18. Comme elle l'indique elle-même dans l'entretien de H. Du Taillis, «Silhouettes de parisiennes, Mlle Léontine Zanta ", La Liberté, 21 juin 1914, p. 2.

19. Concernant ce prix, S. Ducas, "Le prix Femina: la consécration littéraire au féminin ", Recherches féministes, vol. 16, $\mathrm{n}^{\circ} 1,2003, \mathrm{p}$. 43-95.

20. L. Zanta, La science et l'amour, journal d'une étudiante, Paris, Plon, 1922 et La part du feu, Paris, Plon, 1927.

21. R. von Kulessa, Entre la reconnaissance et l'exclusion : la position de l'autrice dans le champ littéraire en France et en Italie à l'époque 1900, Paris, Honoré Champion, 2011.

22. E. Constans, Ouvrières des lettres, Limoges, Presses universitaires de Limoges, 2007, p. 18.

23. C. Lécuyer, «Une nouvelle figure de la jeune fille sous la III ${ }^{e}$ République : l'étudiante », Clio. Histoire, femmes et sociétés, $\mathrm{n}^{\circ} 4,1996$. L'auteure montre l'émergence de cette figure sous le Second Empire, mais sa consolidation entre les années 1900-1930, les étudiantes représentant $12 \%$ des effectifs des étudiants en 1910 et $28 \%$ autour de 1935.

24. F. Blum, "Cent ans de combat pour le droit des femmes ", Après-demain, $\mathrm{n}^{\text {os }} 403-404$, avrilmai 1998, p. 45.

25. Ibid., p. 170.

26. Ibid., p. 117.

27. Ibid., p. 122.

28. Ibid., p. 118.

29. L. Zanta, « La Femme électeur », Le Figaro, 20 décembre 1923, p. 1.

30. L. Zanta, « La femme française réclame ses droits », Le Figaro, 10 mai 1925, n 130.

31. « Radio », L'Ouest Éclair, 18 février 1927, p. 3.

32. L. Zanta, « La Femme électeur », Le Figaro, 20 décembre 1923, p. 1.

33. Comme indiqué dans "Les états généraux du féminisme", Ambassades et consulats : revue de diplomatie internationale, février 1929.

34. Voir S. Aragon, Des liseuses en péril, Paris, Honoré Champion, 2003.

35. Entretien avec Léontine Zanta, "Le rôle de la femme française après la guerre", La renaissance politique, économique, littéraire et artistique, 14 avril 1917, p. 10. Cet entretien est réalisé dans le cadre d'une série d'entrevues sur ce thème organisées par le journal La Renaissance et commencées le 31 mars 1917.

36. F. Thébaud, Écrire l'histoire des femmes et du genre, Paris, ENS Éditions, 2007, p. 196. Plus exactement, comme le rappellent par ailleurs Florence Rochefort et Laurence Klejman, en 1870, la chute de la Commune "prive le féminisme de plusieurs militantes actives, parmi les plus révolutionnaires" (L. Klejman et F. Rochefort, L'égalité en marche. Le féminisme sous la III République, Paris, Presses de la Fondation nationale des sciences politiques, 1989, p. 8). L'existence d'un féminisme socialiste qui s'affirme difficilement a été étudiée par Joan Scott à travers la figure de Madeleine Pelletier, qui termine ses jours, internée en hôpital psychiatrique (J. Scott, La citoyenne paradoxale : les féministes françaises et les droits de l'homme, Paris, Albin Michel).

37. L. Zanta, « La Femme électeur », Le Figaro, 20 décembre 1923, p. 1.

38. Ibid., p. 1.

39. L. Zanta, Psychologie du féminisme, Paris, Plon, 1922.

40. Ibid., p. 5.

41. Ibid.

42. F. Rochefort, "Chapitre II. Féminisme, laïcité et engagements religieux », Associations laïques et confessionnelles. Identités et valeurs, Paris, L'Harmattan, 2006, p. 35-52.

43. C. Bard, "La crise du féminisme en France dans les années trente », Les cahiers du CEDREF, $\mathrm{n}^{\text {os }} 4-5,1995$, p. 13-27.

44. Ibid. 
45. A. Baudrillart, «La question des étudiantes et le devoir des catholiques ", Le Correspondant, 1912, p. 10.

46. B. Poucet, "L'enseignement ", dans B. Duriez, É. Fouilloux, D. Pelletier et N. Viet-Depaule (éds), Les catholiques dans la République, 1905-2005, Paris, Les Éditions de l'Atelier, 2005, p. 121-130.

47. L. Zanta, «Esprit laïque, esprit de destruction », L'Écho de Paris, 2 décembre 1924, p. 1.

48. Ibid., p. 1.

49. L. Zanta, « À la Sorbonne, un regard en arrière », L'Écho de Paris, 20 juillet 1928, p. 1.

50. B. Poucet, "Laïcité et enseignement de la philosophie», Spirale. Revue de recherches en éducation, $\mathrm{n}^{\circ}$ 39, Laïcité, croyances et éducation, 2007, p. 81-91.

51. L. Fedi, "Lien social et religion positiviste chez les penseurs de la Troisième République ", Revue des sciences philosophiques et théologiques, t. 87, 2003, p. 129.

52. Y. Verneuil, «L'école et la laïcité, de l'Ancien Régime à nos jours : enjeux du passé, enjeux dépassés? », Tréma, n 37, 2012, p. 130-143.

53. J. Fabiani, Les philosophes de la République, Paris, Les Éditions de Minuit, 1988, p. 21.

54. L. Zanta, « Diotime et le féminisme », Les Nouvelles littéraires, 30 juillet 1927, p. 2.

55. L. Zanta, Sainte Odile, Paris, Flammarion, 1931 ; Id., Sainte Monique et son fils. La mère chrétienne, Paris, Plon, 1941.

56. F. Muel-Dreyfus, Vichy et l'éternel féminin. Contribution à une sociologie politique de l'ordre des corps, Paris, Seuil, 1996.

57. Voir G. Sapiro, La guerre des écrivains 1940-1953, Paris, Fayard, 1999.

58. C. Cleret, "De la charité à la politique: l'engagement féminin d'Action française», Parlement [s], Revue d'histoire politique, vol. 19, n 1, 2013, p. 17-29.

59. A. Simonin, « La femme invisible : la collaboratrice politique », Histoire@Politique, vol. 9, nº 3, 2009, p. 96.

60. R. Garric, « Le Père Teilhard et Mademoiselle Zanta », Lettres à Léontine Zanta, Paris, Desclée de Brouwer, 1965, p. 16.

61. Ibid.

62. L. Zanta, «L'esprit de haine ", Écho de Paris, 8 avril 1937, p. 1 et 6.

63. «Mlle Léontine Zanta et sainte Odile ", L’Action française, 16 juillet 1931, p. 4.

64. L. Zanta, « Hygiène et art ", L'Écho de Paris, 26 avril 1929, p. 1.

65. Voir D. Lackerstein, National Regeneraiton in Vichy France: Ideas and Policies, 1930-1944, Londres, Routledge, 2012.

\section{RÉSUMÉS}

Cet article met en lumière le parcours saisissant de cette pionnière qu'a été Léontine Zanta, première femme française docteure en philosophie. L'article entend plus particulièrement examiner cette figure à travers le prisme de sa relation à la littérature : car si c'est bien le succès philosophique qui la porte d'abord au statut de pionnière, c'est ensuite sous une forme littéraire, notamment par le roman, que Léontine Zanta poursuit son œuvre. Et Léontine Zanta invente un nouveau personnage littéraire : celui de l'étudiante en philosophie.

L'obiettivo di questo articolo è di mettere in luce il percorso avvincente della pioniera che fu Léontine Zanta, prima donna francese a ottenere il titolo di dottore in filosofia. L'articolo esamina in modo particolare questa figura attraverso il prisma del suo rapporto con la 
letteratura: se fu infatti il successo filosofico che le conferì lo statuto di pioniera, si deve tuttavia sottolineare che Léontine Zanta scelse in seguito la forma letteraria, e soprattutto il romanzo. E Léontine Zanta inventò un nuovo personaggio letterario: la studentessa di filosofia.

This paper highlights the striking career of Léontine Zanta, the first French woman to obtain a doctorate in philosophy. More specifically, the essay examines this figure through the prism of her relationship to literature: although she acquired her status as a pioneer through her early philosophical enterprise, one should stress that Zanta later chose the literary form, and wrote mainly novels. She also invented a new literary character: the philosophy student.

\section{INDEX}

Mots-clés : femmes philosophes, histoire des pionnières, champ littéraire féminin

Parole chiave : donne filosofe, storia delle pioniere, campo letterario femminile

Keywords : women philosophers, history of women pioneers, women's literary field

\section{AUTEUR}

\section{ANNABELLE BONNET}

EHESS, Paris

annabelle.bonnet7@orange.fr 